\title{
The Status of the Heavy Ion Telescope on the ISS/JEM-EF
}

\author{
By Haruka Ueno, ${ }^{1)}$ Haruhisa Matsumoto, ${ }^{1)}$ and Kiyokazu KogA ${ }^{1)}$ \\ 1) Aerospace Research and Development Directorate, JAXA, Tsukuba, Japan
}

(Received June 23rd, 2017)

\begin{abstract}
JAXA has operated the heavy ion telescope located on the ISS/JEM-EF since 2009, and prepared for the publication of data. Due to the long-term operation, there are concerns over the degradation of its silicon sensors and circuits. The calibration operations were conducted in May 2012 and August 2016. We checked for such deterioration but have not yet to confirm the degree of degradation. For the correct determination of atomic number and incident energy, a more accurate calculation method was examined and then applied. As a result, a good correlation with CREME96 was confirmed.
\end{abstract}

Key Words: ISS-JEM, SEDA-AP, Heavy Ions, Galactic Cosmic Ray

\section{Nomenclature}

$$
\begin{array}{ll}
E & : \text { energy } \\
x & : \text { path length } \\
m & : \text { mass of particles } \\
Z & : \text { atomic number }
\end{array}
$$

\section{Introduction}

Although heavy ions coming from solar flares and galactic cosmic rays are not high in flux, they have high linear energy transfer (LET). These particles greatly influence the radiation exposure in manned activities and the single event effects in satellites. In order to measure the heavy ion environment at the ISS altitude, JAXA has operated the Heavy Ion Telescope (HIT), which is one of the sensors of Space Environment Data Acquisition equipment Payload (SEDA-AP) mounted on the Kibo Exposed Facility. ${ }^{1)}$

We plan to disclose the HIT data on our website - the Space Environment Effect System (SEES). ${ }^{2)}$ The distribution of LET, an atomic number, and its incident energy will be open to the public. For LET, the value after Al shielding is considered. It is necessary to verify the integrity of the HIT data in order to publish it. We evaluate the degradation induced by long-term operation for the circuit system and the silicon sensors.

\section{Overview of HIT}

The HIT is a particle identifier telescope, which measures $\mathrm{Li}$ to $\mathrm{Fe}$ ions whose energy is about $10-100 \mathrm{MeV} / \mathrm{n}^{3)}$ The particle identifier telescope consists of a transmission-type detector $(\Delta \mathrm{E}$ detector) and a stop-type detector ( $\mathrm{E}$ detector). The peak value from the $\Delta \mathrm{E}$ detector is proportional to $d E / d x$ for high energy particles. $d E / d x$ is depends on a mass and an atomic of incident particle according from the Bethe-Bloch equation represented by Eq. (1) ${ }^{4)}$ as follows:
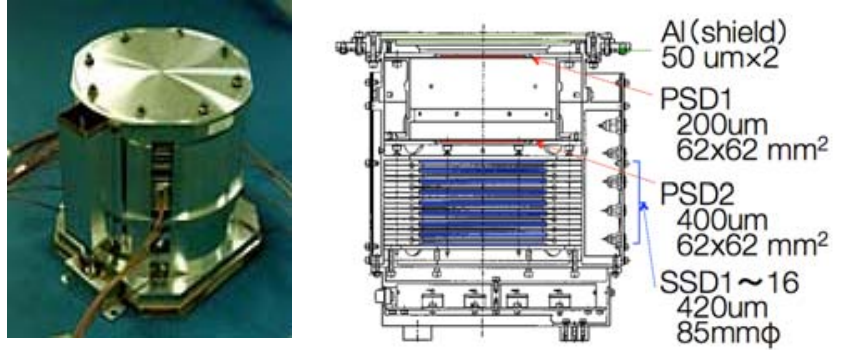

Fig. 1. Photograph (left) and schematic view (right) of the HIT.

$$
\frac{d E}{d x} \propto-\frac{m Z^{2}}{E} .
$$

Figure 1 shows a photograph and a sectional view of the HIT. Eighteen silicon sensors are stacked under two $50-\mu \mathrm{m}$-thick aluminum windows. The top two silicon sensors are position detectors (PSD1 and PSD2), making it possible to correct the energy loss by determining the incident angles. The other sixteen $420-\mu \mathrm{m}$-thick silicon sensors (SSDs) measure the energy loss. Given the inadequate accuracy of measuring the energy loss of PSDs, we assign SSD1 as the $\Delta \mathrm{E}$ detector and SSD1 - SSD15 as the E detector.

As shown in the functional block diagram (Fig. 2), the HIT has an Electric part (HIT-E) and a Sensor part (HIT-S). Measurement begins when energy is measured at PSD1 and PSD2 and the energy losses of all SSDs are read. With energy resolution of 16 bits, the PSDs output values in two systems of high and low gain, respectively, and energy resolution can be changed by incident energy.

\section{Degradation Evaluation}

\subsection{HIT-E}

A circuit for calibration is built in HIT-E. Fig. 3 shows the calibration input signals. High gain is a rectangular wave, and low gain is a triangular wave. For high gain and low gain, 2 and 28 signal lines are assigned, respectively. The degradation 


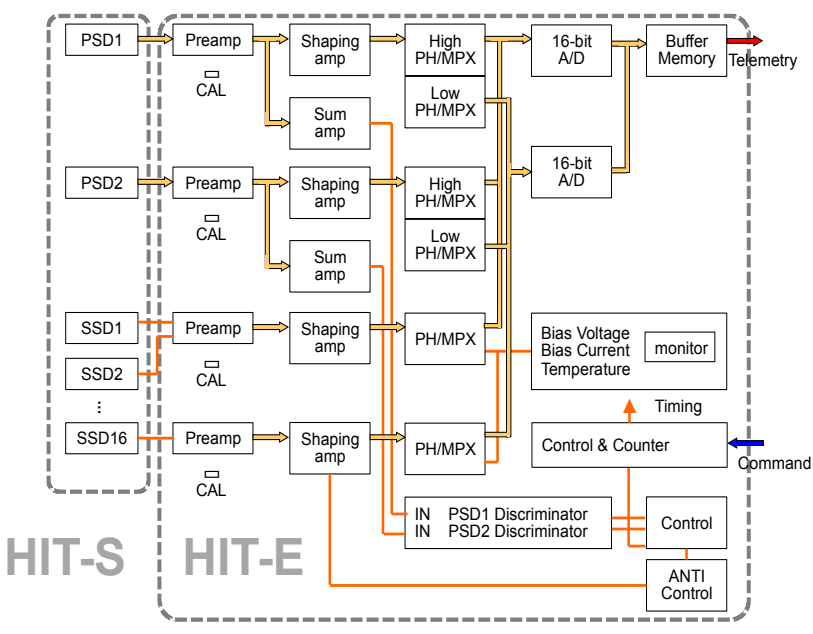

Fig. 2. Block diagram of the HIT function.

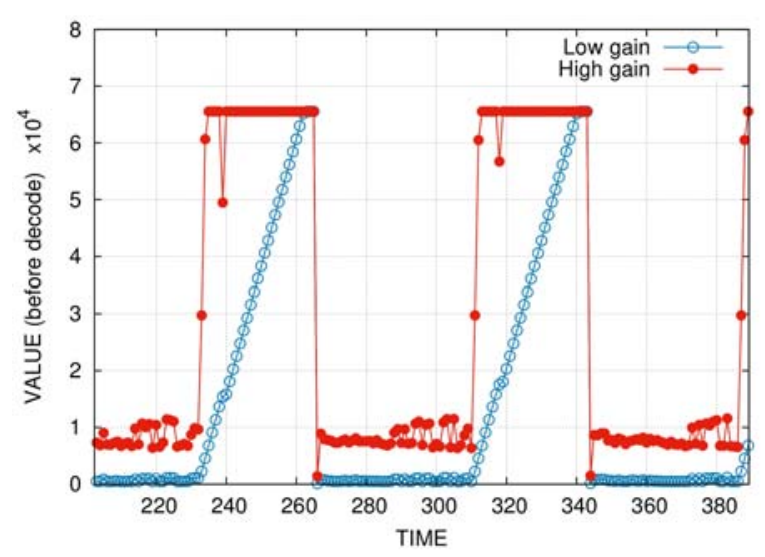

Fig. 3. The calibration signals of Low Gain (circle) and High Gain (filled circle).

of HIT-E is evaluated by changes in the output value for the line assigned at the time of design.

In addition to the initial operation, calibration operations were conducted in May 2012 and August 2016. An evaluation of whether the voltage value of the calibration signal is within the allowable range for all sensors found that all values were within the allowable range in 2012, but in 2016 the high gain of PSD2 exceeded the range. Fig. 4 shows histograms of the voltage value of PSD 2 high gain signals. Approximately 10\% of all signals were out of tolerance in 2016. Whether a problem exists in the trigger signal generation circuit or the signal processing circuit cannot be evaluated. As an influence, since the PSD2 is used as a trigger signal, there is a possibility that trigger malfunction may occur due to an increase or decrease of the signal. However, since energy evaluation is performed by SSD, there is no problem for data analysis.

\subsection{HIT-S}

The degradation of sensors on orbit is generally evaluated using a radiation source, but since one is not installed in HIT, another method is needed. As explained in Section 2, the energy loss in the $\Delta \mathrm{E}$ detector depends on the atomic number.

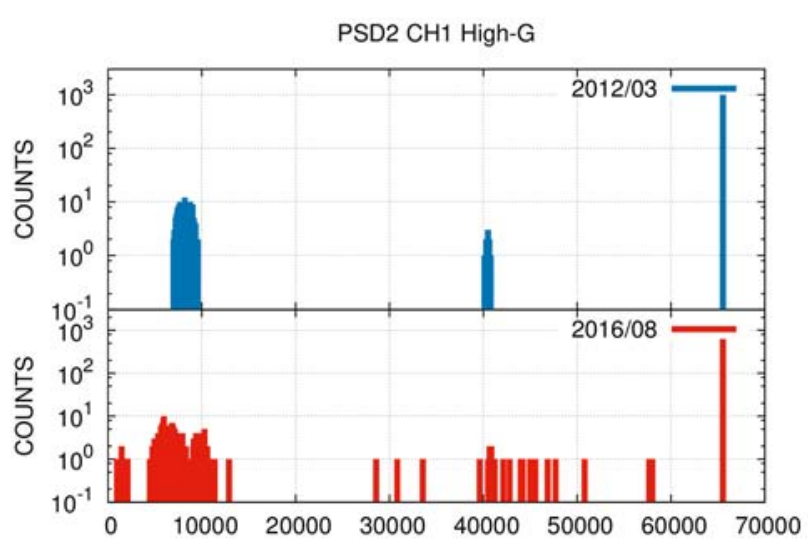

Fig. 4. The case of PSD2 where the allowable range is from 35990 to 46263. The data of 2016 is unstable and some data are out of range.

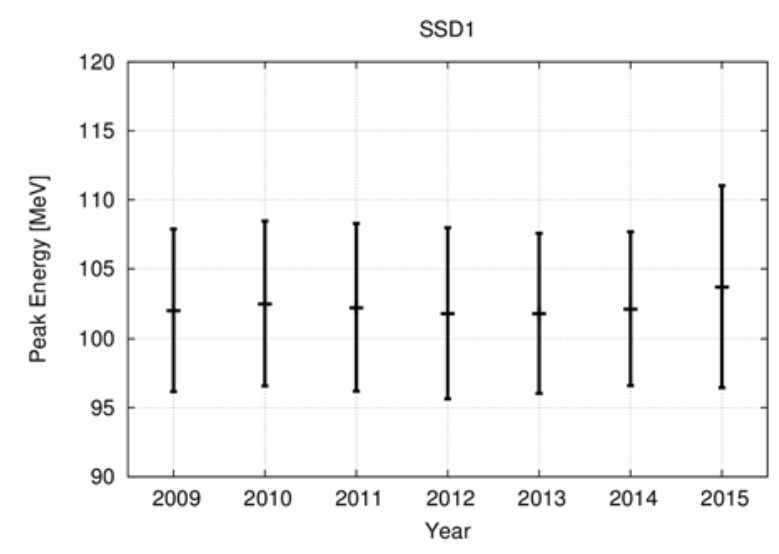

Fig. 5. Time variation of the center value of the Fe-peak from 2009 to 2015 of SSD1. The error bars represent the $90 \%$ confident level.

For an event that is sufficiently high enough to penetrate SSD16, a peak appears for each atomic number in the energy loss histogram. The degradation of a silicon sensor is evaluated by changes in the peak value and width.

We approximate the Fe-peak of the reference with the Gaussian function and obtain its center value. Fig. 5 shows the result and the time variation of SSD1, for instance. The reference peak coincided with the range of the $90 \%$ confidence level, and the degradation of all silicon sensors was not confirmed.

\section{Evaluation of the Z-determination Technique}

\subsection{Method}

Figure 6 shows a scatter plot of all events except noise events. The lines represent the value by simulation. Due to contamination from high energy particles, we omit the events under carbon from this analysis.

Figure 7 indicates an example with carbon. The atomic numbers are provisionally determined by comparing the simulated value and the observed value. It was fitted with the power law function for each provisional atomic number since total energy and deposit energy are in the relation of a power 


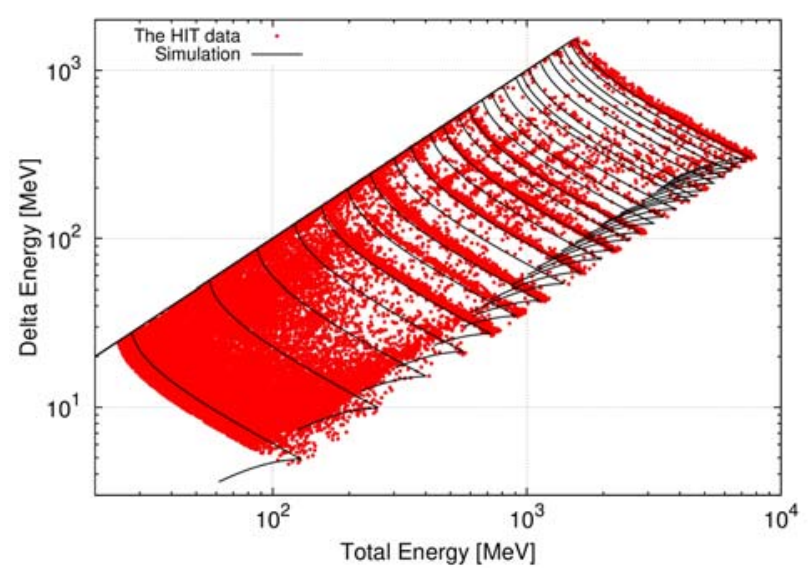

Fig. 6. The scatter plot of all events of the HIT (red-points). The lines represent the value by simulation from $\mathrm{He}$ to $\mathrm{Fe}$ ions.
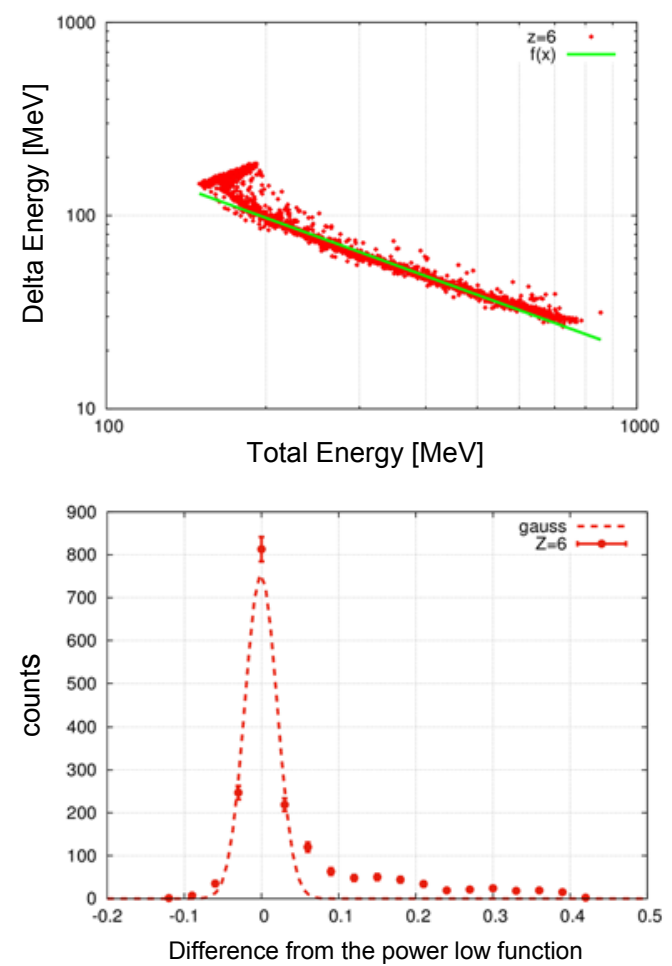

Fig. 7. The case of carbon. The top shows the total energy and deposit energy scatter plot and approximation curve; the bottom shows a histogram of the difference between the fitting curve and observation points.

law. There is a tendency for data points to deviate from the approximate curve at low total energy. It is assumed that these events stop at the very surface of the sensor, attributable to the large ambiguity involved. The bottom panel of Fig. 7 shows a histogram of the difference between the fitting curve and the observation points. We determined that the atomic number is correctly determined for events falling within the 3 sigma of the histogram.

\subsection{Comparison with CREME96}

This process applies for $6 \leqq Z \leqq 26$. To verify the validity of the data, we compare the abundance ratio with a galactic cosmic ray model. Note that the absolute number is smaller than that of the galactic cosmic ray model, because we select

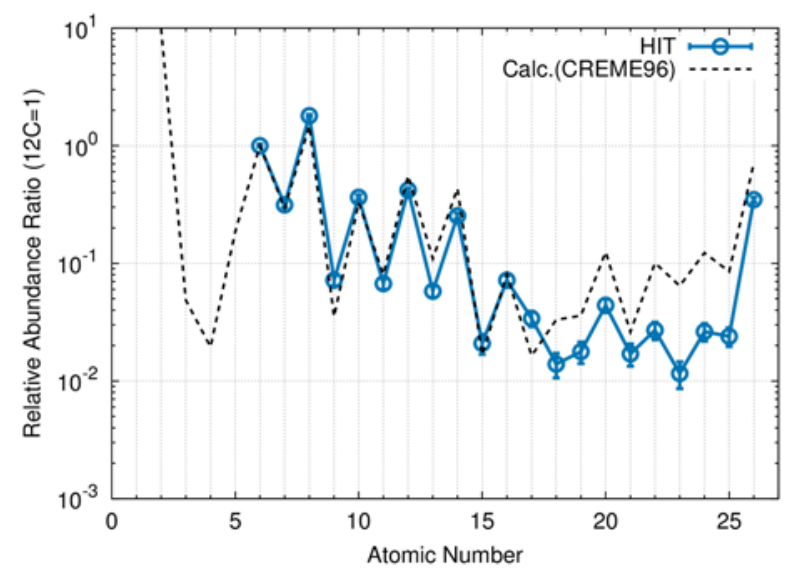

Fig. 8. Comparison between the HIT data (circle) and CREME96 (dotted line) on relative abundance. The HIT data and CREME96 are almost corresponding.

data to find the atomic numbers more accurately. The comparison data was calculated from CREME96. ${ }^{5-7)}$

The comparison of a relative abundance ratio between the HIT data and CREME96 is represented in Fig. 8. Considering the determination accuracy of atomic number, we exclude the data within the period during which pile-up due to solar flare may have occurred. There is a difference from CREME96 when $15<Z<25$, but with other atomic numbers, it coincides with CREME96. Therefore, the correctness of this data processing method was verified.

\section{Conclusion}

For the disclosure of the HIT data in our website the SEES, we examined the evaluation of degradation and the method of determining atomic numbers.

The circuit system and sensor system of the SEDA-AP/HIT were evaluated for deterioration due to aging by using the data of the calibration operations and the energy loss distribution of piercing particles, respectively. No change was observed in the sensor system. The data obtained from the calibration signal voltages of PSD2 in 2016 are unstable than that in 2012. However it can be said that there is no analytical problem since PSD2 is not used for energy measurements. Since degradation due to long-term operation has appeared, it is necessary to perform calibration operation on a regular basis and continuously investigate the soundness of data.

We have newly devised and applied an accurate method of determining atomic numbers. It was confirmed that the calculated element abundance ratios agree well with the galactic cosmic ray model, and that the atomic number can be correctly determined.

\section{Acknowledgments}

We wish to thank Prof. Takashima for his advice on the atomic number determination method. 


\section{References}

1) Koga, K., Matsumoto, H., Obara, T., Kimoto, Y., Yamada, N., Watanabe, H., Endo, M., Sakoh, D., Matsueda, T., Yamamoto, T. , and Muraki, Y.: Initial Result of Space Environment Data Acquisition Equipment-Attached Payload (SEDA-AP) on the ISS Kibo Exposed Facility, Proceedings of RASEDA-9, 2010.

2) Space Environment \& Effects System, JAXA, 2010, http://sees proxy.tksc.jaxa.jp/fw/dfw/SEES/index.html (accessed September 15, 2016)

3) Ueno, H., Matsumoto, H., and Nagamatsu, A.: Measurements from The Heavy-Ion Telescope at The ISS Kibo Exposed Facility, Trans. JSASS Aerospace Tech. Japan, 14, ists30 (2016), pp.67-72.

4) Goka. T., and Matsumoto, H.: High Energy Particle Environment, Review of the Communications Research Laboratory, 43 (1997), pp.249-256 (in Japanese)
5) Tylka, A. J., Adams Jr., J. H., Boberg, P. R., Brownstein, B., Dietrich, W. F., Flueckiger, E. O., Flueckiger, E. L., Petersen, M. A., Shea, D. F., Smart, and Smith, E. C.: CREME96: A revision of the cosmic ray effects on micro-electronics code, IEEE Transactions on Nuclear Science, 44 (1997), pp.2150-2160.

6) Weller, R., Mendenhall, M. H., Reed, R., Schrimpf, R. D., Warren, K. M., Sierawski, B. D. and Massengill, L. W.: Monte Carlo simulation of single event effects, IEEE Transactions on Nuclear Science, 57 (2010), pp.1726-1746.

7) Mendenhall, M. H. and Weller, R. A.: A probability-conserving cross-section biasing mechanism for variance reduction in Monte Carlo particle transport calculations, Nuclear Instruments and Methods in Physics Research Section A: Accelerators, Spectrometers, Detectors and Associated Equipment, 667 (2012), pp.38-43. 